\title{
Pharmacological interventions for those who have sexually offended or are at risk of offending
}

\author{
Omer Khan ${ }^{1}$, Michael Ferriter ${ }^{2}$, Nick Huband ${ }^{3}$, and Nadja Smailagic ${ }^{2}$ \\ ${ }^{1}$ Department of Psychiatry, The Wells Road Centre, Nottingham, UK. \\ ${ }^{2}$ Literature and Evidence Research Unit (LERU), Institute of Mental Health, Nottinghamshire \\ Healthcare NHS Trust, Woodbeck, UK. \\ ${ }^{3}$ Section of Forensic Mental Health, Institute of Mental Health, Nottingham, UK
}

\begin{abstract}
This is the protocol for a review and there is no abstract. The objectives are as follows:

To evaluate the effects of pharmacological interventions on target sexual behaviour for people who have been convicted or at risk of sexual offending.
\end{abstract}

\section{BACKGROUND}

\section{Description of the condition}

Sexual offending is a legal construct which overlaps, but is not entirely congruent with, the clinical constructs of disorders of sexual preference as described in the ICD-10

Classification of Mental and Behavioural Disorders (WHO 1992) or paraphilias as described in the Diagnostic and Statistical Manual of Mental Disorders (Fourth EditionRevised) (APA 1994). Most but not all sexual offences are disorders of sexual preference and most but not all disorders of sexual preference are sexual offences. For instance, clinically defined sexual behaviours such a paedophilia, voyeurism, frotteurism, exhibitionism, zoophilia and necrophilia also meet the rubric for sexual offences but, for instance, fetishism and transvestic fetishism do not, in many jurisdictions. Crimes such as rape, and incest with consenting adult participants are not of themselves classified as disorders of sexual preference or paraphilias.

Copyright () 2009 The Cochrane Collaboration. Published by John Wiley \& Sons, Ltd.

Contact address: Omer Khan, Department of Psychiatry, The Wells Road Centre, The Wells Road, Nottingham, NG3 3AA,

UK.Omer.Khan@nottshc.nhs.uk.

Editorial group: Cochrane Developmental, Psychosocial and Learning Problems Group.

Publication status and date: New, published in Issue 3, 2009.

CONTRIBUTIONS OF AUTHORS: OK was responsible for the clinical background to the protocol and MF was responsible for the methodology section of the protocol. Jo Abbott will run the searches. Two authors (NS and NH) will inspect the identified citations for inclusion and exclusion with a third adjudicating where there is disagreement (OK). Full papers of the included citation will be obtained and reviewed by two authors (MF, NS) with a third reviewer adjudicating (OK) if there is disagreement. Data extraction will be carried out by two reviewers ( $\mathrm{OK}$ and $\mathrm{MF}$ ) with a third adjudicating $(\mathrm{NH})$. Risk of bias tables will be completed by two reviewers $(\mathrm{OK}, \mathrm{NH})$ with a third adjudicating (NS). Data entry will be completed by NS and checked by MF. OK will be responsible for writing the discussion and conclusions.

DECLARATIONS OF INTEREST: None known. 
Sexual offending is both a social problem and a public health issue (Laws 1999). People who have committed a sexual offence represent nearly one quarter of the total population in state prisons in the USA (McGrath 2003). Victim surveys illustrate high incidence and prevalence levels and it is commonly accepted that there is a high proportion of hidden sexual victimization (Chapman 2004,Edwards 2003, Hood 2002). A number of surveys have reported high levels of psychiatric morbidity in survivors of sexual offences (McCauley 1997, Mchichi 2004, Molnar 2001, Swanston 2003,Chapman 2004, Hill 2000).

Sexual offending has therefore become a major challenge for social policy. The electorate and the media expect policy makers to be accountable for the effects of their responses to sexual offending. In the UK, provisions in the Sexual Offences Act 2003 include substantial increases in sentence length for many sexual offences and increased state control, in terms of notification requirements and supervision, for up to ten years after a sentence has been spent (Great Britain 2003). In the USA, such concerns led to Megan's Law in 1996, which allows private and personal information on those registered as sex offenders against children to be made available to the community (About Megan's 2007). Exaggerating the danger that sexual offenders pose is problematic and may increase public fear, stigmatise and hinder rehabilitation of offenders who have changed their lifestyles, while wasting valuable resources on unnecessary surveillance (Soothill 2000).

Quantifying the prevalence of sexual offending is problematic because of variations in definitions of what constitutes sexual abuse, changes in true rates over time and between cultures and countries, and cultural and age related variations in the survivors willingness to disclose abuse. Quantifying its consequences is problematic where an association is not necessarily proof of a causative relationship.

\section{Description of the intervention}

A companion review of psychological interventions for those who have sexually offended or are at risk of offending is currently being updated (Bilby 2008) and it is important to note the underlying difference between psychological and the anti-libidinal approaches with which this review is concerned (see below). The objective of psychological interventions is, in general, to change the sexual behaviour of the offender while leaving libido intact, principally, though not exclusively, from child to adult sexual partners and/or from nonconsenting to consenting sexual activity. In contrast anti-libidinal interventions such as the pharmacological interventions reviewed here seek to greatly diminish or altogether eradicate sexual desire and capacity (Abel 2000). Surgical approaches to anti libidinal interventions such as orchiectomy/orchidectomy (male castration) or neurosurgery will be the subject of a separate review.

Medication-Anti-libidinal medications broadly fall within two categories, namely those medications that have a testosterone suppressing effect, and those medications that affect libido through other mechanisms

The three main classes of testosterone suppressing drugs (TSDs) used today include progestogens, anti-androgens, and Gonadotropin releasing Hormone (GnRH) analogues. 
Prior to the 1960s, oestrogens were prescribed in North America to treat sexually aggressive men but this practice has been discontinued (Grubin 2008).

The commonly used TSDs include Medroxyprogesterone acetate (MPA), Cyproterone acetate (CPA), Triptorelin and Goserelin. Medications which affect libido through means other than via testosterone-suppression, includes antipsychotics and serotonergic antidepressants (SSRI) (Baldwin 2003) and these medications are the most commonly used for this purpose. SSRIs have been associated with reduced libido and delayed orgasm in 60-70\% of people taking them (Montejo 2001).

\section{How the intervention might work}

1.1 Testosterone suppressing drugs-Testosterone production in men is controlled by the hypothalamus, anterior pituitary and the gonads. Gonadotrophin releasing hormone $(\mathrm{GnRH})$ is released from the hypothalamus in a pulsatile manner which stimulates the secretion of the gonadotrophins, Luteinizing Hormone and Follicle Stimulating Hormone (LH and FSH), from the anterior pituitary. LH acts on Leydig cells in the testes resulting in the production and secretion of testosterone which in turn has a negative feedback effect on the anterior pituitary and hypothalamus.

Testosterone has been linked to sexual development and drive in man (Grubin 2008). Testosterone has both organisational and activational effects on the nervous system. The former is associated with structural development of the brain and the latter with the effect of testosterone on the organised brain (Sisk 2006). The effect of testosterone on sexual interest and arousal appears to be one of maintaining a spontaneous level of functionality as opposed to being stimulus bound (Bancroft 1989). These effects are not immediate and take several weeks to occur after a change in plasma testosterone levels (Bancroft 2005).

The direct relationship between testosterone and sexual behaviour is, however, confounded by the link between testosterone and aggressive behaviour (Book 2001). In addition, there is evidence that behaviour may itself result in changes in plasma testosterone levels and that testosterone levels may be more closely associated with dominance (Mazur 1980).

GnRH analogues appear to be more effective than CPA and MPA in producing long term castration levels of testosterone (McEvoy 1999). However, there are few if any studies addressing the issue of reversibility after long term use of greater than two years. GnRH analogues and MPA have been shown to produce histological changes, such as reduced Leydig cell numbers, in the testes of animals (McEvoy 1999, Rao 1998, Avari 1992).

All three agents are associated with potential adverse effects Reilly 2000 such as precipitation or aggravation of cardiac conditions (de Voogt 1986, Pierce 1995). CPA has been associated with fatal liver toxicity (Roila 1993, Parys 1991).

An established consequence of low testosterone, osteoporosis, (Schot 2006, Stepan 1989), is a potentially serious and difficult to manage side effect of testosterone suppression, and is more likely to occur with long term use of GnRH agonists. Other side effects of testosterone 
suppressing drugs include hot flushes, feminisation, depression, weight gain and gynaecomastia (enlargement of male breast tissue) (Grubin 2008).

1.2 Drugs which decrease libido via mechanisms unrelated to testosterone suppression-Although the exact mechanisms are unclear, animal studies suggest that mesolimbic dopamine has an activating effect on sexual arousal and that serotonin has the opposite effect (Hull 2004). In addition, dopamine antagonism may result in prolactin elevation which may have an effect on libido (Bancroft 2005).

Both classes of medication in this category, although widely used, have potentially troublesome side effects. The adverse effects of antipsychotics include tardive dyskinesia (Kane 2006), weight gain (Allison 2003), and impaired glucose tolerance (Haddad 2004) SSRIs are generally considered to have a more benign side effect profile. However, SSRIs are associated with restlessness, agitation, and suicidallity, particularly in the under 30 age group (CSM Working Group 2004) and they are also associated with an increased risk of bleeding (Paton 2005 ).

\section{Why it is important to do this review}

Both the prevalence of sexual offending and the association between victimisation and subsequent mental health problems means that these offences make a significant contribution to mental health morbidity. There is therefore strong social and political pressure to address this problem, not just in terms of helping the victims but in preventing sexual recidivism (Hanson 2000).

An early Cochrane review (White 1998) looked at all types of interventions for sexual offending; it was partially updated in 2003 in a review which considered only psychological interventions (Kenworthy 2003). The older review identified only one small trial $(n=21)$ that compared anti-androgen medication (MPA) plus a psychological intervention versus the same psychological intervention and identifiable no significant difference between the two groups (McConaghy 1988). The reviewers concluded that there were too few data to come to any conclusion about the effectiveness of MPA.

Adi 2002 reviewed the clinical effectiveness and cost-consequences of selective serotonin reuptake inhibitors (SSRIs) in the treatment of sex offenders but identified no RCTs and only nine case series with a total of 225 participants. The authors concluded that there was insufficient data of high enough quality to come to any conclusion.

Subsequently, a systematic review by Lösel and Schmucker (Lösel 2005) was produced which, like that by White et al, considered all treatment options for sex offenders but included studies of a larger range of designs. They reported a treatment effect size of 3.08 (95\% CI 1.40, 6.79, $\mathrm{p}<0.01)$ for anti-libidinal medication based on six identified studies. However, the review does not report sample sizes or study designs for pharmacological interventions and the methodology of the included studies were unclear. There is therefore, an urgent need for an up to date systematic review. 


\section{OBJECTIVES}

To evaluate the effects of pharmacological interventions on target sexual behaviour for people who have been convicted or at risk of sexual offending.

\section{METHODS}

\section{Criteria for considering studies for this review}

Types of studies-Randomised controlled trials with or without blinding will be included. Quasi-randomised trials, such as those where allocation was undertaken on surname, will be excluded.

Types of participants-Adults aged 18 years old and over treated in institutional (prison or psychiatric facility) or community settings for sexual behaviours that have resulted in conviction or caution for sexual offences, offences with a sexual element or violent behaviours with a sexual element (e.g. sexual offences where murder is the index offence), or seeking treatment on a voluntarily basis for behaviours which would be classified as illegal.

We will include studies where behaviours which would be classified as illegal, but where there has been no criminal case reported but where the perpetrator sought treatment or admitted to the behaviours in self report.

Defining what constitutes a sexual offence in the context of the international literature can be problematic as definitions of criminally sexual behaviour differ between jurisdiction, cultures and over time. We will include trials of interventions where the participants have committed a sexual offence which would be accepted by most jurisdictions as crimes, viz. penetrative or non penetrative sexual acts carried out by adults on non consenting adult victims and penetrative or non penetrative sexual acts carried out by adults on consenting or non consenting minors, or adults unable. to give informed consent due to their physical and/or mental disability.

Excluded: Studies of interventions for sex offenders where there is no clear international consensus as to whether the sexual behaviour is a crime or not. Examples include consenting same sex acts between adults, consenting sadomasochistic acts and transvestitism. We will also exclude interventions for sex offenders with learning disability as this is the subject of a separate Cochrane review (Ashman 2008).

Types of interventions-Pharmacological interventions with placebo or standard care (which might include psychological interventions or no treatment).

\section{Types of outcome measures}

Primary outcomes: Recidivism as measured by reconviction, caution, or self report.

Secondary outcomes: Suicide or suicide attempts.

Sudden and unexpected death by other causes. 
Side effects.

Leaving treatment early.

Lost to follow up.

Outcomes will be divided into immediate (within 6 months), short term ( $>6$ months - 24 months) and medium term (>24 months - 5 years) and long term (beyond 5 years) during the period at risk e.g. post release from prison or discharge from hospital facility. If the participants were receiving treatment in the community then the period at risk commences from the end of treatment.

\section{Search methods for identification of studies}

Electronic searches-The following databases will be searched:

Cochrane Central Register of Controlled Trials

MEDLINE

AMED- Allied and Complementary Medicine

ASSIA - Applied Social Sciences Index and Abstracts

BHI - British Humanities Index

Biosis Previews - Biological Abstracts

CINAHL

COPAC - Consortium of University Research Libraries joint catalogue

Dissertation Abstracts

EMBASE

IBSS - International Bibliography of the Social Sciences

ISI Proceedings

ISI-SCI - Science Citation Index Expanded

ISI-SSCI - Social Sciences Citation Index

National Criminal Justice Reference Service Abstracts Database

Open SIGLE

PsycINFO;

Social Care Online 
Sociological Abstracts

UK Clinical Research Network Portfolio Database

ZETOC;

The following strategy will be used to search MEDLINE and modified, where necessary, for the other databases:

\section{1. $\exp$ Sex Offenses/}

2. exp Paraphilias/

3. exp Sexual Behavior/

4. exp Child Abuse, Sexual/

5. exp "Fetishism (Psychiatric)"/

6. exp Exhibitionism/

7. $\exp$ Voyeurism/

8. exp Pedophilia/

9. exp Sadism/

10. exp Masochism/

11. exp Incest/

12. exp Rape/

13. ( $\operatorname{sex} \$ \operatorname{adj} 2$ devia $\$) . t w$.

14. (public adj2 masturbat\$).tw.

15. (child $\$$ adj2 molest\$).tw.

16. (child\$ adj2 (sex\$ or abuse\$)).tw.

17. (sex $\$$ adj2 (murder $\$$ or tortur $\$$ or abus $\$$ or fondl $\$$ )).tw.

18. (indecen $\$$ adj2 behav\$).tw.

19. (child\$ adj2 porn\$).tw.

20. (lewd $\$$ adj2 (behav\$ or act)).tw.

21. bondag\$.tw.

22. frotteur\$.tw.

23. necrophi $\$ . t w$.

24. bugger\$.tw.

25. molest $\$$.tw.

26. pederast $\$$.tw. 
27. paedoph $\$$.tw.

28. pedoph $\$$.tw.

29. scatologia.tw.

30. necrophilia.tw.

31. zoophilia.tw.

32. coprophilia.tw.

33. urophilia.tw.

34. partialism.tw.

35. klismaphilia.tw.

36. bestiality.tw.

37. sodom $\$ . t w$.

38. molest $\$$.tw.

39. paraphil\$.tw.

40. voyeur\$.tw.

41. or $/ 1-40$

Randomised control filters will also be used where appropriate. No language or date restrictions will be applied. The electronic searches will be constructed taking into account changing terminology and perception of sex offences. We recognise that several of these terms would now be regarded as unacceptable and/or misleading as terms signifying sexual offending. These searches are also being run for a related review (Bilby 2008).

\section{Searching other resources}

Hand searching: Reference lists of included studies will be searched for additional relevant trials along with the reference lists of reviews.

Requests for additional data: The first author of each included study and known experts in the field will be contacted for information regarding unpublished data and/or ongoing studies.

\section{Data collection and analysis}

Selection of studies-Titles and abstracts of studies identified through searches of electronic databases will be independently read and assessed by two reviewers (NH, MF). Full copies of those papers which appear to meet the inclusion criteria will then be assessed independently by the two reviewers. We will resolve uncertainties concerning the appropriateness of studies for inclusion in the review through consultation with a third reviewer (OK). Reviewers will not be blinded to the name(s) of the study author(s), their institution(s) or publication sources at any stage of the review. 
Data extraction and management-Data will be extracted independently by two authors (MF \& NH) using a piloted data extraction form. Information on study design and implementation, setting, sample characteristics, intervention characteristics and outcomes will be extracted from all included studies.

Data will be entered into RevMan 5. Where data are not available in the published trial reports, we will contact the authors and ask them to supply the missing information.

Assessment of risk of bias in included studies-For each included study, two authors (NS and HJ) will independently complete the Cochrane Collaboration's tool for assessing risk of bias. This will assess the degree to which:

- the allocation sequence was adequately generated ('sequence generation')

- the allocation was adequately concealed ('allocation concealment')

- knowledge of the allocated interventions was adequately prevented during the study ('blinding')

- incomplete outcome data were adequately addressed

- reports of the study were free of suggestion of selective outcome reporting

Measures of treatment effect-All outcomes are dichotomous and we will use the risk ratio (RR) with a 95\% confidence interval to summarize results within each study. Comparisons will be made at specific follow-up periods:

- within the first six months

- between six and twenty four months

- between 24 and five years

- Beyond five years

Unit of analysis issues-Crossover trials may have been undertaken in this area. The method used to utilize data from any crossover trials will be that recommended in the Cochrane Handbook (Higgins 2008) of approximated paired analysis by imputing missing standard deviations.

Dealing with missing data-The original authors will be contacted for any missing data. However, if the data is still unforthcoming and the investigators have not reported conducting their analysis on an intention-to-treat basis, then we will consider recommendations from the Cochrane Handbook (Higgins 2008) for methods for dealing with missing data.

Where the missing data can be assumed to be missing at random (for example, if there is reason to suppose from included studies that participants left the study because they moved from the area or through other evidence that the effects of the intervention were not related to 'drop outs') we will analyse the available data as presented. 
Where it cannot be assumed that the data is missing at random we may attempt to impute the missing data with replacement values, whether by 'last observation carried forward' (if the assessment points are not far apart in time) or by using other methods to impute the missing data accounting for the fact that these were imputed with uncertainty and using statistical models to allow for missing data. We will seek the advice of a statistician on which methods of of dealing with missing data are appropriate on a case by case basis.

Assessment of heterogeneity-We will assess the extent of between-trial differences and the consistency of results of any meta-analysis in three ways:

- by visual inspection of the forest plots,

- by performing the Chi squared test of heterogeneity (where a significance level less than 0.10 will be interpreted as evidence of heterogeneity)

- by examining the $\mathrm{I}^{2}$ statistic (Higgins 2008; section 9.5.2) The $\mathrm{I}^{2}$ statistic describes approximately the proportion of variation in point estimates due to heterogeneity rather than sampling error. Because heterogeneity may well prove to be a significant problem we will carry out each analysis twice using fixed and random effects models.

Assessment of reporting biases-Funnel plots (effect size versus standard error) will be drawn if sufficient studies are found. We recognize that symmetry of the plots may indicate publication bias, although they may also represent a true relationship between trial size and effect size. If such a relationship is identified, we will examine the clinical diversity of the studies as a possible explanation (Egger 1997).

Data synthesis-We will synthesis data using both a fixed effect and a random effects model.

Meta-analysis will be performed where studies are considered to have sufficiently similar participants, interventions, comparators and outcome measures. Seperate meta-analyses will be carried out for separate classes of pharmacological interventions and separate metaanalyses will also be carried where there are different comapartors e.g. placebo, standard care or psychological interventions. In carrying out meta-analysis, the weight given to each study will be the inverse of the variance so that the more precise estimates (from larger studies with more events) are given more weight.

Subgroup analysis and investigation of heterogeneity-If sufficient studies are found, we will undertake subgroup analysis by type of offending, severity of offending, conviction vs self report.

Sensitivity analysis-We will use sensitivity analysis to assess the impact of study quality if there sufficient data are identified, particularly to consider the potential impact of outcome assessors being blinded, or other factors identified in the risk of bias analysis.

We will also undertake a sensitivity analysis to investigate the robustness of the overall findings where there has been uncertainty or disagreement regarding, for example, the 
inclusion of studies, data extraction or missing data, or in the event of one or more large studies dominating the results.

\section{Acknowledgments}

We would like to thank the authors of both the original reviews by White et al and Kenowrthy et al for establishing the methodology on which this review is principally based. We would like to thank Mark Fenton for devising the search strategy and Lynn Turner and Jo Abbott for running the searches. We would also like to thank Professor Don Grubin and Richard Kreuger for advice on the biological effects of testosterone and testosterone suppressing medications.

\section{SOURCES OF SUPPORT}

\section{Internal sources}

- Nottinghamshire Healthcare NHS Trust, UK.

External sources

- $\quad$ NHS Cochrane Collaboration Programme Grant Scheme, UK.

\section{HISTORY}

Protocol first published: Issue 3, 2009

\section{Additional references}

Abel 2000 . Abel, GG.; Candice, CA. The paraphilias. In: Gelder, MG.; Lopez-Ibor, JJ., Jr; Andreasen, NC., editors. New Oxford Textbook of Psychiatry. Vol. Vol. 1. Oxford University Press; Oxford: 2000.

About Megan’s 2007 . About Megan’s Law. [accessed August 2008] Megan’s Law. 2007. http:// www.about-megans-law.com/

Adi 2002 . Adi Y, Ashcroft D, Browne K, Beech A, Fry-Smith A, Hyde C. Clinical effectiveness and cost-consequences of selective serotonin reuptake inhibitors in the treatment of sex offenders. Health Technology Assessment. 2002; 6(28):1-66. [PubMed: 12583819]

Allison 2003 . Allison DB, Mentore JL, Heo M, Chandler LP, Cappelleri JC, Infante CM, Weiden PJ. Antipsychotic-induced weight gain: a comprehensive research synthesis. American Journal of Psychiatry. 2003; 156:1686-96. [PubMed: 10553730]

APA 1994 . American Psychiatric Association. Diagnostic and Statistical Manual of Mental Disorders (DSM-IV). American Psychiatric Association; Washington DC: 1994.

Ashman 2008 . Ashman L, Duggan L. Interventions for learning disabled sex offenders. Cochrane Database of Systematic Reviews. 2008; (Issue 1) [DOI: 10.1002/14651858.CD003682.PUB2].

Avari 1992 . Avari KM, Bhiwgade DA. Effect of depot medroxyprogesterone acetate and testosterone anathate on the testis of albino rats: ultrastructural and biochemical studies. Indian Journal of Experimental Biology. 1992; 30:1118-27. [PubMed: 1294476]

Baldwin 2003 . Baldwin D, Mayers A. Sexual side effects of antipsychotic and antidepressant drugs. Advances in Psychiatric Treatment. 2003; 9:202-10.

Bancroft 1989 . Bancroft, J. Human Sexuality and its Problems. Churchill Livingstone; Edinburgh: 1989.

Bancroft 2005 . Bancroft J. The Endocrinology of Sexual Arousal. Journal of Endocrinology. 2005; 10:411-27. [PubMed: 16135662]

Bilby 2008 . Bilby C, Ferriter M, Jones H, Huband N, Smailagic N. Psychological interventions for those who have sexually offended or are at risk of offending. Cochrane Database of Systematic Reviews. 2008; (Issue 4) [DOI: 10.1002/14651858.CD007507.].

Book 2001 . Book AS, Starzyk KB, Quinsey VL. The relationship between testosterone and sexual aggression: A meta-analysis. Aggression and Violent Behaviour. 2001; 6:579-99. 
Chapman 2004 . Chapman DP, Whitfield CL, Felitti VJ, Dube SR, Edwards VJ, Anda RF. Adverse childhood experiences and the risk of depressive disorders in adulthood. Journal of Affective Disorders. 2004; Vol. 82:217-25. [PubMed: 15488250]

CSM Working Group 2004 . Committee on Safety of Medicines (CSM). [accessed May 2009] Expert working group on the safety of selective serotonin reuptake inhibitor antidepressants. 2004. http://www.mhra.gov.uk/home/groups/plp/documents/websiteresources/con019470.pdf

de Voogt 1986 . de Voogt HJ, Smith PH, Pavone-Macaluso M, de Pauw M, Suciu S. Cardiovascular side effects of diethylstilbestrol, cyproterone acetate, medroxyprogesterone acetate and estramustine phosphate used for the treatment of advanced prostatic cancer: results from European Organization for Research on Treatment of Cancer trials 30761 and 30762. Journal of Urology. 1986; 135:303-7. [PubMed: 2935644]

Edwards 2003 . Edwards VJ, Holden GW, Felitti VJ, Anda RF. The relationship between multiple forms of childhood maltreatment and adult mental health in community respondents: results from the adverse childhood experience study. American Journal of Psychiatry. 2003; Vol. 160(issue 8):1453-9. [PubMed: 12900308]

Egger 1997 . Egger M, Davey-Smith G, Schneider M, Minder C. Bias in meta-analysis detected by a simple, graphical test. British Medical Journal. 1997; (issue 315):629-34. [PubMed: 9310563]

Great Britain 2003 . Great Britain. Sexual Offences Act 2003 Elizabeth II. The Stationery Office; London: 2003. Chapter 42. Accessible online at: http://www.opsi.gov.uk/Acts/acts2003/ ukpga_20030042_en_1[ISBN 9780 105442035] [accessed August 2008]

Grubin 2008 . Grubin, D. Medical Models and Interventions in Sexual Deviance. In: Laws, DR.; O'Donohue, W., editors. Sexual Deviancy: Theory, Assessment and Treatment. 2nd Edition. The Guildford Press; New York: 2008.

Haddad 2004 . Haddad PM. Antipsychotics and diabetes: review of non-prospective data. British Journal of Psychiatry. 2004; 185(Supplement):S80-S86.

Hanson 2000 . Hanson RK, Harris AJ. Where should we intervene? Dynamic predictors of sexual offense recidivism. Criminal Justice and Behavior. 2000; 27:6-35.

Higgins 2008 . Higgins, JPT.; Green, S., editors. Cochrane Handbook for Systematic Reviews of Interventions. Version 5.0.0. The Cochrane Collaboration; 2008. Available from http:// handbook.cochrane.org/ [Updated February 2008]

Hill 2000 . Hill J, Davis R, Byatt M, Burnside E, Rollinson L, Fear S. Childhood sexual abuse and affective symptoms in women: a general population study. Psychological Medicine. 2000; Vol. 30:1283-91. [PubMed: 11097069]

Hood 2002 . Hood R, Shute S, Feilzer M, Wilcox A. Sex offenders emerging from long-term imprisonment: A study of their long-term reconviction rates and of parole board members 'judgements of their risk'. British Journal of Criminology. 2002; 42(2):371-94.

Hull 2004 . Hull EM, Muschamp JW, Sato S. Dopamine and Serotonin: Influences on male sexual behaviour. Physiology and Behaviour. 2004; 83:291-307.

Kane 2006 . Kane JM. Tardive Dyskinesia circa 2006. American Journal of Psychiatry. 2006; 163:1316-18. [PubMed: 16877639]

Kenworthy 2003 . Kenworthy T, Adams CE, Bilby C, Brooks-Gordon B, Fenton M. Psychological interventions for those who have sexually offended or are at risk of offending. Cochrane Database of Systematic Reviews. 2003; (Issue 4) [DOI: 10.1002/14651858.CD004858].

Laws 1999 . Laws R. Harm reduction or harm facilitation: A reply to Maletsky. Sexual Abuse: A Journal of Research and Treatment. 1999; 11:233-40. [PubMed: 10497782]

Lösel 2005 . Lösel F, Schmucker M. The effectiveness of treatment for sexual offenders: A comprehensive meta-analysis. Journal of Experimental Criminology. 2005; Vol. 1(issue 1):11746.

Mazur 1980 . Mazur A, Lamb T. Testosterone and dominance in men. Behavioural and Brain Sciences. 1980; 21:353-63.

McCauley 1997 . McCauley J, Kern DE, Kolodner K, Dill L, Schroeder AF, DeChant HK, et al. Clinical characteristics of women with a history of childhood abuse. JAMA. 1997; Vol. 277(issue 17):1362-8. [PubMed: 9134941] 
McConaghy 1988 . McConaghy N, Blaszczynski A, Kidson W. Treatment of sex offenders with imaginal desensitization and/or medroxyprogesterone. Acta Psychiatrica Scandinavica. 1988; 77:199-206. [PubMed: 2896429]

McEvoy 1999 . McEvoy, G., editor. AHFS Drug Information. American Society of Health System Pharmacists; Bethesda, Maryland: 1999.

McGrath 2003 . McGrath RJ, Cumming G, Livingston JA, Hoke SE. Outcome of a treatment program for adult sex offenders. Journal of Interpersonal Violence. 2003; 18(1):3-17.

Mchichi 2004 . Mchichi AK, Kadri N. Moroccan women with a history of child sexual abuse and its long-term repercussions: A population-based epidemiological study. Archives of Womens Mental Health. 2004; Vol. 7:237-42.

Molnar 2001 . Molnar BE, Buka SL, Kessler RC. Child sexual abuse and subsequent. American Journal of Public Health. 2001; Vol. 91(issue 5):753-60. [PubMed: 11344883]

Montejo 2001 . Montejo AL, Llorca G, Izquierdo JA, Rico-Villademoros F. Incidence of sexual dysfunction associated with antidepressant agents: a prospective multicenter study of 1022 outpatients. Journal of Clinical Psychiatry. 2001; 62(Supplement 3):10-21. [PubMed: 11229449]

Parys 1991 . Parys BT, Hamid S, Thomson RG. Severe hepatocellular dysfunction following cyproterone acetate therapy. British Journal Urology. 1991; 67:312-30.

Paton 2005 . Paton C, Ferrier IN. SSRIs and gastrointestinal bleeding. BMJ. 2005; 331:529-30. [PubMed: 16150746]

Pierce 1995 . Pierce EJ. Angina pectoris and myocardial infarction with the use of leuprolide acetate. American Journal of Obstetrics and Gynecology. 1995; 172(4 Pt. 1):1323. [PubMed: 7726282]

Rao 1998 . Rao MV, Roy GK, Prasannalata SN. Effect of medroxyprogesterone acetate and testosterone enanthate on vas deferens of rats. Indian Journal of Experimental Biology. 1998; 36:157-61. [PubMed: 9754044]

Reilly 2000 . Reilly DR, Delva NJ, Hudson RW. Protocols for the use of cyproterone, medroxyprogesterone, and leuprolide in the treatment of paraphilia. Canadian Journal of Psychiatry. 2000; 45:559-563.

Roila 1993 . Roila F, Crino L, Carloni G, Natalini G. Cyproterone acetate: hepatotoxicity and prostate cancer treatment. Annals of Oncology. 1993; 4:701. [PubMed: 8241005]

Schot 2006 . Schot LPC, Schuurs AHWM. Pathophysiology of bone loss in castrated animals. The Journal of Steroid Biochemistry and Molecular Biology. 1990; 37:461-5. [PubMed: 2257251]

Sisk 2006 . Sisk CL. New insights into the neurobiology of sexual maturation. Sexual and Relationship Therapy. 2006; 21:5-14.

Soothill 2000 . Soothill K, Francis B, Sanderson B, Ackerley E. Sex offenders: specialists, generalists or both? A 32-year criminological study. British Journal of Criminology. 2000; 40:56-67.

Stepan 1989 . Stepan JJ, Lachman M, Zverina J, Pacovsky V, Baylink DJ. Castrated men exhibit bone loss: effect of calcitonin treatment on biochemical indices of bone remodelling. Journal of Clinical Endocrinology and Metabolism. 1989; 69:523-7. [PubMed: 2788171]

Swanston 2003 . Swanston HY, Plunkett AM, O’Toole BI, Shrimpton S, Parkinson PN, Oates RK. Nine years after child sexual abuse. Child Abuse and Neglect. 2003; Vol:27-967.

White 1998 . White. Managements for people with disorders of sexual preference and for convicted sexual offenders. Cochrane Database of Systematic Reviews. 1998; (Issue 4) [Art. No.: CD000251. DOI: 10.1002/14651858.CD000251].

WHO 1992 . WHO. The ICD-10 Classification Of Mental and Behavioural Disorders. World Health Organization; Geneva: 1992.

* Indicates the major publication for the study 